\title{
A DECOMPOSITION OF ADDITIVE SET FUNCTIONS ${ }^{1}$
}

\author{
R. S. PHILLIPS
}

This paper is concerned with a decomposition theorem for additive functions on an additive family of sets to either real numbers or a Banach space. Additive bounded set functions have as yet been little studied. However the recent paper of Hildebrand $t^{2}$ illustrates their importance.

We shall use the following notation:

(a) $T$ : an abstract class of arbitrary elements $t$.

(b) J: a completely additive family of subsets $\tau$ of $T$; that is, $T \varepsilon$ J, $\tau \varepsilon \mathfrak{J}$ implies $T-\tau \varepsilon \mathfrak{J}$, and $\tau_{n} \varepsilon \mathfrak{J}$ for $n=1,2, \ldots$ implies $\sum \tau_{n} \varepsilon \mathfrak{J}$.

(c) $\alpha$ : a set function on $J$ to real numbers.

(d) $A$ : the subclass of set functions on $J$ to real numbers which are additive and bounded; that is, $\tau_{1}, \tau_{2} \varepsilon J$ and $\tau_{1} \cdot \tau_{2}=0$ implies $\alpha\left(\tau_{1}+\tau_{2}\right)=\alpha\left(\tau_{1}\right)+\alpha\left(\tau_{2}\right)$.

(e) $C$ : the subclass of set functions on $J$ to real numbers which are completely additive (c.a.), that is, $\tau_{n} \varepsilon \Im$ for $n=1,2, \cdots$ and $\tau_{i} \cdot \tau_{j}=0$ if $i \neq j$ implies $\alpha\left(\sum \tau_{n}\right)=\sum \alpha\left(\tau_{n}\right)$. The functions in $C$ are bounded. ${ }^{3}$

The notations $A_{P}$ and $C_{P}$ refer to the subclasses of $A$ and $C$ respectively whose elements are nonnegative.

(f) $x$ : a set function on $J$ to a Banach space $X$. The definitions of additive and c.a. set functions are formally retained. If $\left\{\tau_{n}\right\}$ is a sequence of disjoint sets of $J$ and $x(\tau)$ is c.a., then $\sum x\left(\tau_{n}\right)$ is unconditionally convergent. ${ }^{5}$

(g) $C_{X}$ : the class of c.a. set functions on 3 to $X$.

In the statement of the following theorems, $D$ will designate any one of the classes $A, A_{P}, C, C_{P}$, and $\bar{\tau}$ will denote the cardinal number of $\tau$.

THEOREM 1. Let $\aleph$ be an infinite cardinal number not greater than $\overline{\bar{T}}$. For every $\alpha$ \& $D$ there exists an unique decomposition $\alpha=\alpha_{1}+\alpha_{2}$ and a set $R(\alpha) \varepsilon \Im$ of cardinal number not greater than $\aleph$ such that $\alpha_{1}, \alpha_{2} \varepsilon D$,

1 Presented to the Society April 15, 1939, under the title On additive set functions.

$2 \mathrm{~T}$. H. Hildebrandt, On bounded linear functional operations, Transactions of this Society, vol. 36 (1934), pp. 868-875.

${ }^{3}$ S. Saks, Theory of the Integral, Monografje Matematyczne, Warsaw, 1937, p. 10, Theorem 6.1.

${ }^{4} \mathrm{~S}$. Banach, Théorie des Opérations Linéaires, Monografje Matematyczne, Warsaw, 1932, chap. 5 .

${ }^{5}$ If $x_{n}$ is a series of elements of $X$ and if every subseries $\sum x_{n}$ is convergent, then $\sum x_{n}$ is said to be unconditionally convergent. 


$$
\alpha_{1}(\tau)=\alpha(R \cdot \tau), \quad \alpha_{2}(\tau)=0 \quad \text { if } \quad \bar{\tau} \leqq \aleph .
$$

Let $\Sigma \equiv E_{\tau}[\tau \varepsilon \Im, \bar{\tau} \leqq \aleph, \alpha(\tau) \neq 0]$. We define a transfinite sequence $\left(\tau_{1}, \tau_{2}, \cdots ; \tau_{\omega}, \cdots, \tau_{\lambda}, \cdots\right)$ as follows: $\tau_{1}$ is an arbitrary element of $\Sigma$. Suppose $\tau_{\lambda}$ have been defined for all $\lambda<\mu$. If there exists $\tau$ such that $\tau \cdot \sum_{\lambda<\mu} \tau_{\lambda}=0$ and $\tau \varepsilon \Sigma$, then we set $\tau=\tau_{\mu}$.

As $\alpha(\tau)$ is bounded, $\alpha(\tau)$ cannot differ from zero on a nondenumerable number of disjoint sets. The sequence therefore contains only a denumerable set of elements.

Let $R=\sum_{\lambda} \tau_{\lambda}$. Then $R \varepsilon J$ and $\bar{R} \leqq \aleph$. We define $\alpha_{1}(\tau)=\alpha(R \cdot \tau)$, $\alpha_{2}(\tau)=\alpha(\tau)-\alpha_{1}(\tau)=\alpha(\tau-R \cdot \tau)$. The $\alpha_{1}(\tau), \alpha_{2}(\tau)$ are clearly elements of $D$. If $\bar{\tau} \leqq \aleph$, then by the definition of $R, \alpha_{2}(\tau)=\alpha(\tau-R \cdot \tau)=0$.

Although the set $R$ is not unique, the function decomposition is unique: Suppose there exist two different sets $R_{1}, R_{2}$ having the properties of the $R$ defined above. The set identity $R_{1} \cdot \tau+\left(R_{2}-R_{1}\right) \cdot \tau$ $=R_{2} \cdot \tau+\left(R_{1}-R_{2}\right) \cdot \tau$ and $\alpha\left[\left(R_{1}-R_{2}\right) \cdot \tau\right]=0=\alpha\left[\left(R_{2}-R_{1}\right) \cdot \tau\right]$ imply that $\alpha\left(R_{1} \cdot \tau\right)=\alpha\left(R_{2} \cdot \tau\right)$.

A set function $\alpha$ on $J$ will be said to be nonsingular if for every $t \varepsilon \mathfrak{J}, \alpha(t)=0$. A set function $\alpha$ on $\mathfrak{J}$ will be called $\boldsymbol{\aleph}$-homogeneous if there exists a set $R$ such that $R \varepsilon \mathcal{J}, \bar{R}=\boldsymbol{N}, \alpha(\tau)=\alpha(R \cdot \tau)$, and $\alpha(\tau)=0$ if $\bar{\tau}<\boldsymbol{N}$.

Without loss of generality we may consider only nonsingular set functions because for every $\alpha \varepsilon D$ there exists a unique decomposition $\alpha=\alpha_{1}+\alpha_{2}$ and a denumerable set $\left\{t_{i}\right\}$ of elements of $T$, such that $\alpha_{1}, \alpha_{2} \varepsilon D, \alpha_{1}(\tau)=\sum_{i=1}^{\infty} \alpha\left(\tau \cdot t_{i}\right)$, and $\alpha_{2}$ is nonsingular. We omit the proof.

THEOREM 2. For every nonsingular $\alpha \varepsilon D$, there exists an unique decomposition $\alpha=\sum_{i} \alpha_{i}$, the sum being absolutely convergent, and such that $\alpha_{i}$ is $\boldsymbol{\aleph}_{i}$-homogeneous and $\boldsymbol{\aleph}_{i} \neq \boldsymbol{\aleph}_{j}$ if $i \neq j$.

In the proof of this theorem an induction is made on the infinite cardinals not exceeding that of $T$, well-ordered according to magnitude. We define a transfinite sequence of set functions $\left(\alpha_{1}, \alpha_{2}, \cdots ; \alpha_{\omega}, \cdots, \alpha_{\lambda}, \cdots\right)$ as follows: Suppose $\alpha_{\lambda}$ have been defined for all $\lambda<\mu$ and (1) only a denumerable number of the $\alpha_{\lambda}$ are not identically zero; (2) $\sum_{\lambda \leqq \lambda_{0}}\left|\alpha_{\lambda}(\tau)\right|<\infty$; and (3) $\alpha_{\lambda} \varepsilon D$ and is $\aleph_{\lambda}$-homogeneous. By Theorem 1 there exist $R_{\mu} \varepsilon J$ and a decomposition $\alpha=\alpha_{\mu}^{1}+\alpha_{\mu}^{2}$ such that $\bar{R}_{\mu} \leqq \aleph_{\mu}, \alpha_{\mu}^{1}(\tau)=\alpha\left(R_{\mu} \cdot \tau\right), \alpha_{\mu}^{2}(\tau)=0$ if $\bar{\tau} \leqq \aleph_{\mu}$, and $\alpha_{\mu}^{1}, \alpha_{\mu}^{2} \varepsilon D$. Clearly $\alpha_{\lambda}(\tau)=\alpha\left(R_{\mu} \cdot R_{\lambda} \cdot \tau\right)$ if $\lambda<\mu$.

Let $\alpha_{\mu}(\tau)=\alpha_{\mu}^{1}(\tau)-\sum_{\lambda<\mu} \alpha_{\lambda}(\tau)$. We consider the following cases:

I. $\alpha \varepsilon C, C_{P}$. Let $\bar{R}_{\mu}=R_{\mu}-\sum_{\pi_{\mu}} \bar{R}_{\lambda}$ where $\pi_{\mu} \equiv E_{\lambda}\left[\lambda<\mu, \alpha_{\lambda} \neq 0\right]$. The sets $\bar{R}_{\mu}$ are disjoint. Suppose $\alpha_{\lambda}(\tau)=\alpha\left(\bar{R}_{\lambda} \cdot \tau\right)$ for $\lambda<\mu$. Then by (1) 


$$
\begin{aligned}
\alpha_{\mu}(\tau) & =\alpha\left(R_{\mu} \cdot \tau\right)-\sum_{\pi_{\mu}} \alpha_{\lambda}(\tau)=\alpha\left(R_{\mu} \cdot \tau\right)-\sum_{\pi_{\mu}} \alpha\left(R_{\mu} \cdot \bar{R}_{\lambda} \cdot \tau\right) \\
& =\alpha\left[\left(R_{\mu}-\sum_{\pi_{\mu}} R_{\mu} \cdot \bar{R}_{\lambda}\right) \cdot \tau\right]=\alpha\left(\bar{R}_{\mu} \cdot \tau\right) .
\end{aligned}
$$

It is clear that (1), (2), and (3) are satisfied for $\mu+1 . \alpha_{\lambda} \neq 0$ implies that $\alpha(\tau) \neq 0$ for some subset of $\bar{R}_{\lambda}$. As the $\bar{R}_{\lambda}$ are disjoint, the sequence will contain only a denumerable number of functions not identically zero.

II. $\alpha \varepsilon A_{P}$. For $\lambda_{0}<\mu, \alpha(T) \geqq a_{\lambda_{0}}^{1}(T) \equiv \sum_{\lambda \leqq \lambda_{0}} \alpha_{\lambda}(T) \geqq \sum_{\lambda \leqq \lambda_{0}} \alpha_{\lambda}(\tau)$. Clearly (1) and (2) are satisfied for $\mu+1$, and the sequence contains only a denumerable number of functions not identically zero. Let $\lambda_{i}$ be a spanning sequence for $E_{\lambda}\left[\lambda<\mu, \alpha_{\lambda} \neq 0\right]$. Then

$$
\begin{aligned}
\alpha_{\mu}(\tau) & =\alpha_{\mu}^{1}(\tau)-\sum_{\lambda<\mu} \alpha_{\lambda}(\tau)=\alpha\left(R_{\mu} \cdot \tau\right)-\lim _{i \rightarrow \infty} \alpha_{\lambda_{i}}^{1}(\tau) \\
& =\alpha\left(R_{\mu} \cdot \tau\right)-\lim _{i \rightarrow \infty} \alpha\left(R_{\mu} \cdot R_{\lambda_{i}} \cdot \tau\right) .
\end{aligned}
$$

Hence (3) is likewise satisfied.

III. $\alpha \varepsilon A$. Every $\alpha \varepsilon A$ has a decomposition $\alpha=\alpha_{1}-\alpha_{2}$ where $\alpha_{1}, \alpha_{2} \varepsilon A_{P}$. An application of II to $\alpha_{1}$ and $\alpha_{2}$ gives the desired decomposition.

The decomposition is unique: Any two sequences of homogeneous functions differ in a first function, $\alpha_{\mu}$. But this is contrary to $\alpha_{\mu}^{1}=\sum_{\lambda \leqq \mu} \alpha_{\lambda}$ being unique.

In these theorems the restriction that the additive bounded set function be defined over an additive family $J$ is optional, since the range of definition of such a function can always be extended to an additive family. The type of argument used by Pettis ${ }^{6}$ will prove this statement.

We next consider the possibility of extending these theorems to functions $x(\tau)$ on $J$ to a Banach space. The theorem is not in general valid for additive bounded set functions of this type. This is illustrated by $x(\tau)$ defined on all subsets of $T \equiv(0,1)$ to the space $X$ of bounded functions on $S \equiv(0,1)$ where $x(\tau)$ is the characteristic function of the subset of $S$ which has the same coordinate values as $\tau$. Clearly there exists no denumerable set $R$ such that $x(\tau-R \tau)=0$ for all denumerable sets $\tau$.

However analogous theorems are obtained for c.a. set functions on $\Im$ to $X$.

${ }^{6}$ B. J. Pettis, Linear functionals and completely additive set functions, Duke Mathematical Journal, vol. 4 (1938), p. 554, Theorem 1.1. 
THEOREM 3. Let $\aleph$ be an infinite cardinal number not greater than $\overline{\bar{T}}$. For every $x \& C_{X}$ there exists a unique decomposition $x=x_{1}+x_{2}$ and a set $R(x) \varepsilon J$ of cardinal power not greater than $\aleph$ such that $x_{1}, x_{2} \varepsilon C_{X}$, $x_{1}(\tau)=x(R \cdot \tau), x_{2}(\tau)=0$ if $\overline{\bar{\tau}} \leqq \boldsymbol{N}$.

$x(\tau) \neq 0$ on at most a denumerable number of disjoint sets of 3 . Suppose the contrary. Then there exists a denumerable sequence of disjoint sets $\left\{\tau_{i}\right\}$ and an $e>0$ such that $\left\|x\left(\tau_{i}\right)\right\|>e,(i=1,2, \cdots)$. As $x(\tau)$ is c.a., $\sum_{i} x\left(\tau_{i}\right)$ converges. The supposition is therefore false.

The argument used in Theorem 1 will now prove the theorem.

THEOREM 4. For every nonsingular $x \varepsilon C_{X}$, there exists an unique decomposition $x=\sum_{i} x_{i}$, the sum being unconditionally convergent, and such that $x_{i}$ is $\boldsymbol{\aleph}_{i}$-homogeneous and $\boldsymbol{\aleph}_{i} \neq \boldsymbol{\aleph}_{j}$ if $i \neq j$.

The proof is identical with that of I in Theorem 2. Again there will exist disjoint $\bar{R}_{\mu}$ 's such that $x_{\mu}(\tau)=x\left(\bar{R}_{\mu} \cdot \tau\right)$.

UNIVERSITY OF MichigAN 